\title{
Application of Functional Rehabilitation after Ligament Injury in The Knee Joint Exercise Therapy in Track and Field Athletes
}

\author{
Liu Qiang, XiaoLongling School of Physical Education, Wenshan University, Wenshan Yunnan
} 663099, China

346591653@163.com

\section{Keywords: Exercise therapy; ligament injury; athletes; rehabilitation treatment}

\begin{abstract}
Limb function rehabilitation exercise therapy treatment after exploring athletes' knee ligament injury. Methods January 2013-August 2014 period in our hospital 40 cases of knee injuries Athletes tough as the research object, according to the rehabilitation process, whether to exercise therapy patients were divided into observation group and conventional group 20 cases.Observation group were given exercise therapy, conventional therapy for the conventional group. result : Two grades of limb function, $\mathrm{P}<0.05$, the difference was statistically significant. The treatment group throughout the recovery time was $(10.2 \pm 1.4)$ weeks, significantly shorter than the control group $(14.5 \pm 2.6)$ weeks; treatment group recurrence rate was $5.0 \%$, compared with $15.0 \%$ in the control group, two groups, the differences were there was statistically significant ( $\mathrm{P}$ $<0.05)$. Conclusion Exercise therapy can improve athletes' good after knee ligament injury limb function, should be widely applied.
\end{abstract}

\section{Introduction}

When joints suffer non-physiological activity due to violence, force ligament stretch beyond its tolerance range will be varying degrees of ligament damage. Patients with ligament injury will be local pain, tissue congestion, swelling, tenderness and movement disorder[1-2]. Movement therapy is based neurodevelopmental science, biomechanics, be passive or active through exercise to achieve improved alternative compensatory purposes, and ultimately improve patient injury local blood circulation and metabolism, relieve stress abnormal, further corrective dysfunction, physical deformity[3]. January 2013 in our hospital - to be when 40 cases of knee injuries Athletes rehabilitation period August 2014 study sports therapy, achieved satisfactory results, are reported below.

\section{Materials and Methods}

\subsection{General Information}

Our hospital in January 2013 - August 2014 admitted during tough knee injury in cases of 40 track and field athletes chosen for the study, according to the rehabilitation process, whether to exercise therapy patients were divided into observation group and conventional group 20 cases. Conventional group 13 males, 7 females; aged 15-23 years, mean (21.4 \pm 2.4 ) years; Rating: athlete in 16 cases, 4 cases of a; the site of injury: lateral collateral ligament in 3 cases, 14 cases of medial collateral ligamentposterior cruciate ligament three cases; previous history of trauma in 18 cases. Observation group, 12 males and 8 females; aged 14-26 years, mean $(22.1 \pm 1.8)$ years; Rating: athlete in 14 cases, a six cases;previous history of trauma in 17 cases.Between the two groups of patients gender, age, grade, location and previous history of trauma injury, was not statistically significant $(\mathrm{P}>0.05)$. 


\subsection{Methods}

General conventional therapy group, the observation group shall exercise therapy rehabilitation.

1.2.1 patients with acute training Including: (1) contact quadriceps contraction: contraction 5, relax 2s, each 2 times to complete 200 work; (2) ankle pump training, counter-clockwise alternately, at least $8 \mathrm{~h}$ a day; (3) straight leg raise: min 2 per day times to complete 100 times, each time unable to adhere to the degree; (4) stretch exercise: according to the characteristics of tissue regeneration and repair, after the completion of the fiber repair, if early training, should start using natural, gentle tension, to promote well-organized repair, each lasting about 20s, eight consecutive times, 1/d [4].

1.2.2 function in patients with active training (1) open chain movement: Patients were sitting, hands hold the knee, knees slowly to a maximum degree of pain and insisted 1min, 10 complete set of actions every day; (2) squat: the affected knee flexion greater than $90 \mathrm{He}$ began to practice after ${ }^{\circ}$, squat knees and stick to the maximum reluctantly $1 \mathrm{~min}, 10$ complete set of actions every day; (3) rich in straight leg raise: 2 times a day a total of 100 groups, each exercise is difficult to adhere to for the degree, according to gradually increase the load exercise in ; (4) closed chain exercise: 50 times / d, do ipsilateral knee flexion movement, toes touch the ground firmly placed lunge, heels false step, mainly on the quadriceps and medial head bent to $90^{\circ}$ for exercise [5].

2.2.1 Strength Training of patients Including(1) isokinetic exercise: Early selectively slow or medium speed, in order to facilitate increased muscle tension, promote muscle recovery. After each training session to $60 \% \mathrm{~s}$ as a starting velocity, after each exercise 10 times increase $30 \% \mathrm{~s}$, up to $180 \% \mathrm{~s}$, gradually reduce the $30 \%$ until reduced to $60 \%$, followed by cycle exercise continuous shrinkage of 1100 training units, depending on the patient's condition, can be carried out 3-4 times a week. Late can be high-speed, repeated the second largest contraction training, velocity accessible daily activities, when sports systolic velocity at $300^{\circ} / \mathrm{s}$ or more. Isokinetic exercise capacity of patients with rehabilitation activities, has an important role to return to the stadium. When the affected knee flexor/extensor peak torque ratio of 60\%-80\%, bilateral knee flexors/extensors peak torque ratio can be a special time to exercise can be more than $85 \%$; (2) coordination and balance abilityexercise: exercise can choose when balancing balls, each about 10min, 3-4 times a week; skipping once a day, each lasting about $20 \mathrm{~min}$ to; (3) aerobic exercise: choose ergometer, treadmill exercise, each lasting 45min, 3 times a week; (4) weight-bearing exercises toes: each 10 times, 10 groups/d, 3 times/week; mainly triceps strength exercise;(5) Plyometric contraction exercise: rehabilitation when patients recover better late, can withstand high explosive start exercising, exercise such as jumping steps, each interval $48 \mathrm{~h}$ once, twice a week -3 times before training required to perform warm-up exercise[6-7].

2.2.2 Strength Training of patients Including(1) isokinetic exercise:Early selectively slow or medium speed,in order to facilitate increased muscle tension, promote muscle recovery. After each training session to $60 \% \mathrm{~s}$ as a starting velocity, after each exercise 10 times increase $30 \%$, up to $180 \%$, gradually reduce the $30 \%$ s until reduced to $60 \%$, followed by cycle exercise continuous shrinkage of 100 training 1 units,depending on the patient's condition,can be carried out 3-4 times a week.Late can be high-speed,repeated the second largest contraction training,velocity accessible daily activities, when sports systolic velocity at $300 \%$ s or more. (2) coordination and balance abilityexercise:exercise can choose when balancing balls,each about 10min, 3-4 times a week; skipping once a day,each lasting about 20min to; (3) aerobic exercise:choose ergometer, treadmill exercise,each lasting 45min,3 times a week; (4) weight-bearing exercises toes:each 10 times, 10 groups/d,3 times/week; mainly triceps strength exercise;(5) Plyometric contraction exercise: rehabilitation when patients recover better late, can withstand high explosive start exercising,exercise such as jumping steps, each interval $48 \mathrm{~h}$ once, twice a week-3 times before 
training required to perform warm-up exercise [6-7].

\section{3 determine the efficacy index}

According Brunnstrom evaluation method[2] on the limb function was classified as 1-6, respectively, before the completion of treatment and exercise therapy were assessed one month after the higher the level of physical activity is better explained.

\subsection{Statistical}

Collection and processing of data in this study by the Hospital Data Processing Center specialized personnel in order to ensure data authenticity and scientific. Preliminary data entry EXCEL (2003 Edition) logical proof and analysis; and the use of SPSS22.0 software package for data processing, count data using the $\mathrm{n}(\%)$ that the use $\chi^{2}$ test; using $\bar{x} \pm s$ the measurement data, using $t$ test; the test results with $\mathrm{P}<0.05$ was considered statistically significant.

\section{Results}

3.1 Brunnstrom scores between the two groups, as shown in Table 1.

Table 1 Brunnstrom scores between the two groups $(\mathrm{n}=20)$

\begin{tabular}{ccccccc}
\hline Group & Level 1 & Level 2 & Level 3 & Level 4 & Level 5 & Level 6 \\
\hline Observer Group & 0 & 0 & 0 & 0 & 1 & 18 \\
Group & 0 & 1 & 1 & 3 & 4 & 11 \\
$\chi^{2}$ & & & 2.6651 & \\
P & & & 0.0077 & \\
\hline
\end{tabular}

Note: The two grades of limb function, $\mathrm{P}<0.05$.

3.2 Comparison of the two groups, as shown in Table 2.

Table 2 Comparison of two groups of the general index $(\mathrm{n} ; \%)$

\begin{tabular}{ccccc}
\hline Group & $\begin{array}{c}\text { Number } \\
\text { of cases }\end{array}$ & $\begin{array}{c}\text { Knee flexors / extensors } \\
\text { peak torque ratio of } 60 \% \\
-80 \%\end{array}$ & $\begin{array}{c}\text { Bilateral knee flexors, } \\
\text { extensors peak torque } \\
\text { ratio }>85 \%\end{array}$ & $\begin{array}{c}\text { The rate of } \\
\text { return to the } \\
\text { training field }\end{array}$ \\
\hline Observer Group & 20 & $20(100.0)$ & $19(95.0)$ & $20(100.0)$ \\
Group & 20 & $17(85.0)$ & $17(85.0)$ & $18(90.0)$ \\
t & - & 3.2432 & 1.1111 & 2.1053 \\
P & - & 0.0717 & 0.2918 & 0.1468 \\
\hline
\end{tabular}

\subsection{Two groups of patients recovery time and recurrence results}

The treatment group throughout the recovery time was $(10.2 \pm 1.4)$ weeks, significantly shorter

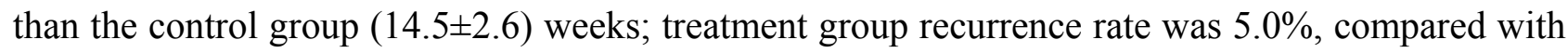
$15.0 \%$ in the control group, two groups, the differences were there was statistically significant $(\mathrm{P}<0.05)$. The results are shown in Table 3 . 
Table 3 sets of results and comparison of recurrence in patients with rehabilitation time

\begin{tabular}{cccc}
\hline Group & $n$ & Recovery time (weeks) & Recurrence rate $(\%)$ \\
\hline Observer Group & 20 & $10.2 \pm 1.4^{*}$ & $5.0^{*}$ \\
Group & 20 & $14.5 \pm 2.6$ & 15.0 \\
$\chi^{2}$ & -- & 1.24 & 2.36 \\
P & -- & $<0.05$ & $<0.05$ \\
\hline
\end{tabular}

Note: Compared with the control group, $* \mathrm{P}<0.05$

\section{Discussion}

After conservative treatment of domestic and foreign scholars have recognized,the early and late fixed exercise is conducive to rehabilitation after ligament injury shaping[8].Currently,most physicians are advocates of early to be controlled early exercise regularly, prompting the patient as soon as possible to repair ligament damage. I understand the premise of conservative treatment is the need for a good team, to the full rehabilitation guidance for athletes,followed by the doctor-patient cooperation, combining static and dynamic, both global and local, while developing the corresponding means of rehabilitation for different injury,continuing to explore and analyze treatment the problem, in order to further enhance the therapeutic effect. In professional sports teams or institutions, usually equipped with such therapists can give some excellent athletes with better rehabilitation to restore to optimal levels. This study shows that two athletes in the Brunnstrom grade comparison, $\mathrm{P}<0.05$, statistically significant; In addition, the observation group athletes to return to the training field was $100 \%, 10 \%$ higher than the conventional group, confirmed that exercise therapy for knee Athletes significant efficacy in the treatment of joint injury. The exercise therapy will help to improve function in patients with limited, well improved patient health status, body functions, minimizing the potential harm after the knee injury.

In short,exercise therapy in the adjuvant treatment of knee ligament injury has played a good role,as long as a good grasp of its safe and effective method of treatment,no significant side effects,worthy of promotion.

\section{References}

[1]Donghui Yang,Quanxia Gu.early rehabilitation in the anterior cruciate ligament reconstruction Arthroscopic treatment of [J] Chinese Journal of General Practitioners, 2012,(7): 532-534.

[2]Jinquan Liu, Chongxia Huang,Weiliang Zhu,etc. massage and physical therapy and other treatment of acute medial collateral ligament injury Clinical Observation on 20 cases [J] Journal of Yunnan Chinese medicine, 2013,34 (5):4-48.

[3] Liang Xue,Bangmeng You,Lijuan Jiao.etc. Movement Therapy in knee medial collateral ligament injury rehabilitation [J] Modern Medicine, 2011,20 (4): 420-421.

[4] Liang Xue, Bangmeng You,Lijuan Jiao. Movement Therapy in knee medial collateral ligament injury rehabilitation [J] Modern Medicine, 2011,20 (4):420-1.

[5] Jinquan Liu,Chongxia Huang, Weiliang Zhu. Massage and physical therapy of acute knee medial collateral ligament injury Clinical Observation on 20 cases [J] Journal of Yunnan Chinese medicine, 2013,5 (34):47-8. 
[6]. WormaldPJ. The agger nasi cell: the key to understanding the anatomy of the frontal recess. Otolaryngol Head Neck Surg. $2011,129: 497-507$.

[7] CHO I B I,,LEE HJ,H AN JK,et al. Detection of hypervascula r nodular hepatocellur carcinomas: value of triphasic helica 1 C T compared with iodized oil C T[ J]..A J R,2 0 10, $157(2): 219-22$ 4

[ 8 ] K H A N A,COM BS CS,,B RUN T EM,,et al.Positron emissi on tomography scanning in the evaluation of hepatocellular carcinoma[J]. Ann Nucl Med,2009,14(2):121-126 